\title{
ON THE VARIATION OF THE ARBITRARY AND GIVEN CONSTANTS
}

\section{IN DYNAMICAL EQUATIONS*}

\author{
BY \\ ERNEST WILLIAM BROWN
}

\section{Introduction.}

The method of the variation of arbitrary constants is a well-known device for obtaining the solution of a set of differential equations when we know that of another set which differs from the former in a suitable manner. Especially is it useful in most of the problems of celestial mechanics where methods of approximation have to be adopted. The first approximation once obtained, it gives the second approximation with ease and certainty, and the same idea can be followed out for the higher approximations if further accuracy is desired.

In the class of problems under consideration here, the equations of motion can be put into the form

$$
\frac{d^{2} x_{i}}{d t^{2}}=\frac{\partial F}{\partial x_{i}} \quad(i=1,2, \cdots n)
$$

or into the form, known as canonical,

$$
\frac{d x_{i}}{d t}=\frac{\partial F}{\partial X_{i}}, \quad \frac{d X_{i}}{d t}=-\frac{\partial F}{\partial x_{i}} \quad(i=1,2, \cdots n),
$$

where $F$ is a function either of the $x_{i}$ or of the $x_{i}, X_{i}$, respectively, and may or may not contain the time explicitly. Suppose that the problem has been solved so that the $x_{i}, X_{i}$ are expressed in terms of $2 n$ arbitrary constants and the time, and that it be required to find the solution of another problem in which $F+R$ replaces $F$. The new problem can be stated by supposing the arbitrary constants in the first problem to be variable and finding the differential equations satisfied by them.

The function $F$ will usually contain, besides $x_{i}, t$, a number of given constants which will naturally be present in the solution, and it may happen that some of these constants are really variables which, in order to get a first approximation, we have treated as constants. It is, of course, not possible in general

* Presented to the Society under a different title April 25, 1903. Received for publication April 20, 1903. 
to solve the equations as though they were constant and then substitute their variable values in the solution. If we follow the method mentioned we may ask what variable values are to be given to the arbitraries in order that the variable values of the given constants may be substituted in the solution. Thus instead of adding $R$ to $F$ the new problem is that obtained by replacing some of the constants present in $F$ by functions of the time; it is chiefly this problem that I propose to consider in the following pages:

In order to do so it is necessary to consider in some detail the relations satisfied by the derivatives of the dependent variables, not only with respect to the arbitraries but also with respect to the given constants. In the course of this investigation, generalizations of certain theorems which I gave in 1897 * (which included generalizations of ADAMs' theorems connecting the mean motions of the perigee and node with the constant term in the expression for the moon's parallax) are obtained and some new relations between the various constants are added ( $\S 1-4)$. In $\S 5$ the equations for the variable values of the arbitraries corresponding to variable values for the given constants are obtained. It there appears that Newcomв's theorem (see art. 24) is a particular case of a general theorem which applies to all equations of dynamics satisfying the conditions laid down. In $\S 6$ applications of the results obtained are made to the lunar theory and in particular to the effects which variable values for the given constants produce on the motions of the arguments.

\section{$\S 1$. The Equations of Variations.}

1. Let $(x, y, z)$ be the coördinates, referred to fixed axes, of a particle moving under a force-function $F$. The equations of motion are

$$
\frac{d^{2} x}{d t^{2}}=\frac{\partial F}{\partial x}, \quad \frac{d^{2} y}{d t^{2}}=\frac{\partial F}{\partial y}, \quad \frac{d^{2} z}{d t^{2}}=\frac{\partial F}{\partial z} .
$$

The solution of these equations involves a certain number of constants : of these, six are arbitrary and the others are known and present in $F$.

Denote by $f, g$ any two of these constants and differentiate (1) with respect to $f, g$ successively. We find

$$
\begin{aligned}
& \frac{d^{2}}{d t^{2}} \frac{d x}{d f}=\frac{\partial^{2} F}{\partial x^{2}} \frac{d x}{d f}+\frac{\partial^{2} F}{\partial x \partial y} \frac{d y}{d \bar{f}}+\frac{\partial^{2} F}{\partial x \partial z} \frac{d z}{d f}+\frac{d^{\prime}}{d f} \frac{\partial F}{\partial x}, \\
& \frac{d^{2}}{d t^{2}} \frac{d x}{d g}=\frac{\partial^{2} F}{\partial x^{2}} \frac{d x}{d g}+\frac{\partial^{2} F}{\partial x \partial y} \frac{d y}{d g}+\frac{\partial^{2} F}{\partial x \partial z} \frac{d z}{d g}+\frac{d^{\prime}}{d g} \frac{\partial F}{\partial x},
\end{aligned}
$$

with similar equations for the other coördinates; the operators $d^{\prime} / d f, d^{\prime} / d g$

* On certain properties of the mean motions, etc., Proceedings of the London Mathematical Society, vol. 28 (1896), pp. 143-155. 
denote derivatives with respect to $f, g$ only in so far as they occur explicitly in $F$.

Multiply these equations by $d x / d g,-d x / d f$ respectively, and add the corresponding results for the other two coördinates. Denoting by $S$ a sum of similar expressions with respect to the three coördinates, we obtain an equation which may be written,

$$
\frac{d}{d t}(f, g)=S\left(\frac{d x}{d g} \frac{d^{\prime}}{d f} \frac{\partial F}{\partial x}-\frac{d x}{d f} \frac{d^{\prime}}{d g} \frac{\partial F}{\partial x}\right)=\frac{d}{d g} \frac{d^{\prime} F}{d f}-\frac{d}{d f} \frac{d^{\prime} F}{d g},
$$

where

$$
(f, g)=S\left(\frac{d x}{d g} \frac{d}{d t} \frac{d x}{d f}-\frac{d x}{d f} \frac{d}{d t} \frac{d x}{d g}\right)=-(g, f)
$$

If $h$ be another of the constants we have, by direct differentiation,

$$
\frac{d}{d f}(g, h)+\frac{d}{d g}(h, f)+\underset{d h}{d}(f, g)=0 .
$$

2. The constants will now be separated into two classes. Suppose that the solution is such that $F$ and each coördinate is expressible by means of sums of cosines or sines of sums of multiples of the angles

$$
b_{i} t+\beta_{i}=w_{i},
$$

where $b_{i}$ and the coefficients of the periodic terms depend only on one set of constants $\alpha_{i}$, the other set being $\beta_{i}$, and the $\alpha_{i}, \beta_{i}$ being all independent of one another; these will be respectively called the $\alpha$-set and the $\beta$-set. It is supposed that $F$ is given in terms of $x, y, z$ and the known constants, involving the latter in the form just given. It is also supposed that the expressions for $F$ and for its derivatives contain no term independent of $x, y, z$. Also the constant of length is to be such a function of the arbitrary constants of the solution that $x, y, z$, the kinetic energy $T$, and $F$ contain no term independent of the arbitraries. In general, we shall avoid choosing the constant of length as an arbitrary so that certain functions which are only defined by their derivatives with respect to the arbitraries need no added constant independent of them.

3. Let $\alpha, \alpha^{\prime}$ be two constants of the $\alpha$-set, and $\beta, \beta^{\prime}$ two of the $\beta$-set corresponding to angles $w, w^{\prime}$. Denote by $\partial / \partial \alpha$ derivatives with respect to $\alpha$ only in so far as $\alpha$ is contained in the coefficients, so that $d / d f$ refers to derivatives when $\alpha_{i}, \beta_{i}$ are considered independent and $\partial / \partial f$ to derivatives when $\alpha_{i}, w_{i}$ are considered independent ; evidently $\partial / \partial w_{i}=\partial / \partial \beta_{i}=d / d w_{i}=d / d \beta_{i}$. 
Denote by $\dot{x}, \dot{y}, \dot{z}$ derivatives with respect to $t$. Since $\dot{x}=\Sigma_{i} b_{i} d x / d \beta_{i}$, we have

$$
\begin{aligned}
\frac{d x}{d \alpha} & =\frac{\partial x}{\partial \alpha}+t \Sigma_{i} \frac{\partial x}{\partial w_{i}} \frac{d b_{i}}{d \alpha} \\
\frac{d}{d t}\left(\frac{d x}{d \alpha}\right) & =\frac{d}{d t}\left(\frac{\partial x}{\partial \alpha}\right)+\Sigma_{i} \frac{\partial x}{\partial w_{i}} \frac{d b_{i}}{d \alpha}+t \Sigma_{i} \frac{\partial \dot{x}}{\partial w_{i}} \frac{d b_{i}}{d \alpha} \\
& =\frac{\partial \dot{x}}{\partial \alpha}+t \Sigma_{i} \frac{\partial \dot{x}}{\partial w_{i}} \frac{d b_{i}}{d \alpha}
\end{aligned}
$$

in which it is to be noted that $\partial / \partial \alpha, d / d t$ are not commutative although $\partial / \partial w_{i}$, $d / d t$ are so.

Substituting these results in (3) and putting

we obtain

$$
[f, g]=S\left(\frac{\partial \dot{x}}{\partial f} \frac{\partial x}{\partial g}-\frac{\partial x}{\partial f} \frac{\partial \dot{x}}{\partial g}\right)=-[g, f]
$$

$$
\begin{aligned}
\left(\alpha, \alpha^{\prime}\right)=\left[\alpha, \alpha^{\prime}\right]+t \Sigma_{i}\left\{\frac{d b_{i}}{d \alpha}\left[w_{i}, \alpha^{\prime}\right]-\frac{d b_{i}}{d \alpha^{\prime}}\left[w_{i}, \alpha\right]\right\} & \\
& +t^{2} \Sigma_{i} \Sigma_{j} \frac{d b_{i}}{d \alpha} \frac{d b_{j}}{d \alpha}\left[w_{i}, \dot{w}_{j}\right],
\end{aligned}
$$

$$
\begin{aligned}
(\alpha, \beta) & =[\alpha, w]+t \Sigma_{i} \frac{d b_{i}}{d \alpha}\left[w_{i}, w\right], \\
\left(\beta, \beta^{\prime}\right) & =\left[w, w^{\prime}\right] .
\end{aligned}
$$

Also

$$
\frac{\partial}{\partial f}[g, h]+\frac{\partial}{\partial g}[h, f]+\frac{\partial}{\partial h}[f, g]=0 .
$$

The quantities $(f, g)$ must be divided into several classes, each of which requires separate consideration. The constants $f, g$ may both belong to the $\alpha$-set, or to the $\beta$-set, or one to the $\alpha$-set and the other to the $\beta$-set. Again, they may both be arbitrary, or both known, or one arbitrary and one known. I shall only consider two known constants - one belonging to each set-so that there will be eight different kinds of brackets to consider, three of them being independent of the known constants. The extension to any number of known constants is made without difficulty.

\section{§2. Application to the Arbitrary Constants.}

4. When the constants considered in $\S 1$ are the arbitraries of the solution, the right hand member of $(2)$ is zero since $F^{\prime}$ does not contain them explicitly; all the $(f, g)$ are therefore constant. But $\left[\alpha, \alpha^{\prime}\right],\left[\beta, \beta^{\prime}\right]$ consist only of 
sums of sines owing to the forms assumed for the coördinates. Hence by $\S 1$, art. 3,

$$
\begin{gathered}
\left(\alpha, \alpha^{\prime}\right)=\left[\alpha, \alpha^{\prime}\right]=\left(\beta, \beta^{\prime}\right)=\left[w, w^{\prime}\right]=0,\left(\alpha, \beta_{i}\right)=\left[\alpha, w_{i}\right] \\
\Sigma_{i} \frac{d b_{i}}{d \alpha}\left[\alpha^{\prime}, w_{i}\right]=\Sigma_{i} \frac{d b_{i}}{d \alpha^{\prime}}\left[\alpha, w_{i}\right] .
\end{gathered}
$$

Also from (6),

hence

$$
\frac{\partial}{\partial \alpha}\left[\alpha^{\prime}, w\right]-\frac{\partial}{\partial \alpha^{\prime}}[\alpha, w]=\frac{\partial}{\partial w}\left[\alpha^{\prime}, \alpha\right]=0 ;
$$

(9) $\left[\alpha_{i}, w_{j}\right]=\left(\alpha_{i}, \beta_{j}\right)=\frac{d c_{j}}{d \alpha_{i}}=\frac{\partial^{2}}{\partial \alpha_{i} \partial w_{j}}\left(c_{1} w_{1}+c_{2} w_{2}+c_{3} w_{3}\right) \quad(i, j=1,2,3)$,

where $c_{1}, c_{2}, c_{3}$ are functions of the arbitrary and known constants of the $\alpha$-set only; they are thus completely defined in accordance with the remark at the end of art. 2.

Equation (8) then gives

$$
\Sigma_{i} \frac{d b_{i}}{d \alpha} \frac{d c_{i}}{d \alpha^{\prime}}=\Sigma_{i} \frac{d b_{i}}{d \alpha^{\prime}} \frac{d c_{i}}{d \alpha}
$$

or

showing that

$$
\frac{d}{d \alpha}\left(\Sigma_{i} b_{i} \frac{d c_{i}}{d \alpha^{\prime}}\right)=\frac{d}{d \alpha^{\prime}}\left(\Sigma_{i} b_{i} \frac{d c_{i}}{d \alpha}\right)
$$

$$
\Sigma_{i} b_{i} d c_{i}=-d B
$$

a perfect differential. This gives a complete definition of $B$ if we assume that it contains no term independent of the arbitrary constants.

5. Canonical systems.-The constants $\alpha_{i}$ can in general be chosen arbitrarily. Take $\alpha_{i}=c_{i}$. Then

$$
\left[c_{i}, w_{i}\right]=\left(c_{i}, \beta_{i}\right)=1, \quad\left[c_{i}, w_{j}\right]=\left(c_{i}, \beta_{j}\right)=0 \quad(j \neq i) .
$$

The choice is possible if the Jacobian of the $c_{i}$ with respect to the $\alpha_{i}$ is not zero ; in the applications to be made it is easy to see that this is the case. Thus the $c_{i}$ are assumed to be independent. We then have from art. 4,

$$
b_{i}=-\frac{d B}{d c_{i}}
$$

where $B$ is expressed in terms of the $c_{i}$ and the known constants.

All these results are perfectly well known. The sets $c_{i}, \beta_{i}$ and $c_{i}, w_{i}$ are canonical systems, for the $(f, g),[f, g]$ are nothing else than LaGrange's brackets and the above values reduce LAGRANGE's equations for the variation 
of arbitrary constants, when a disturbing function $R$ is added to $F$, to the canonical forms

and

$$
\frac{d c_{i}}{d t}=\frac{d R}{d \beta_{i}}, \quad \frac{d \beta_{i}}{d t}=-\frac{d R}{d c_{i}},
$$

$$
\frac{d c_{i}}{d t}=\frac{d R^{\prime}}{d w_{i}}, \quad \frac{d w_{i}}{d t}=-\frac{d R^{\prime}}{d c_{i}}
$$

where $R^{\prime}=R+B$ and $R$ is supposed to be expressed in terms of the $w_{i}, c_{i}, t$ and known constants.

6. Another canonical set is obtained by putting

$$
B_{i}=\frac{d c_{1}}{d \alpha_{i}} \beta_{1}+\frac{d c_{2}}{d \alpha_{i}} \beta_{2}+\frac{d c_{3}}{d \alpha_{i}} \beta_{3}
$$

and taking $\alpha_{i}, B_{i}$ as arbitraries; this is evident from equation (9). The same equation shows that if we put

$$
W_{i}=\frac{d c_{1}}{d \alpha_{i}} w_{1}+\frac{d c_{2}}{d \alpha_{i}} w_{2}+\frac{d c_{3}}{d \alpha_{i}} w_{3},
$$

the $\alpha_{i}, W_{i}$ form a canonical set.

The sets $c_{i}, \beta_{i} ; \alpha_{i}, B_{i} ; \alpha_{i}, W_{i}$ have the disadvantage that when we form the equations of variations the time appears as a factor of the periodic terms. This fact prevents them from being useful for most purposes of the lunar theory to which application will chiefly be made. It is to be noticed nevertheless that when $\beta_{i}$ or $w_{i}$ are taken as one set of arbitraries, the $c_{i}$ is the conjugate set which makes the whole canonical; if $\alpha_{i}$ be taken as one set then the $B_{i}$ or $W_{i}$ has the same property with reference to the $\alpha_{i}$. In general, if one set of constants be given the second set which renders the whole canonical is determined uniquely; the apparent duplicity in the above is due to the fact that $W_{i}$, $w_{i}$ are not arbitrary constants but contain the time.

If it be possible to choose the $b_{i}$ as one set of arbitraries, the conjugate sets are

and from $(10)$,

$$
\Sigma_{j} \beta_{j} \frac{d c_{j}}{d b_{i}}=\Sigma_{j} \beta_{j} \frac{d c_{i}}{d b_{j}}, \quad \Sigma_{j} w_{j} \frac{d c_{j}}{d b_{i}}=\Sigma_{j} w_{j} \frac{d c_{i}}{d b_{j}} \quad(j=1,2,3) ;
$$

so that

$$
c_{i}=\frac{d}{d b_{i}}\left(B+\Sigma_{j} b_{j} c_{j}\right)
$$

$$
\left(b_{i}, \beta_{j}\right)=\left[b_{i}, w_{j}\right]=\frac{d^{2}}{d b_{i} d b_{j}}\left(B+b_{1} c_{1}+b_{2} c_{2}+b_{3} c_{3}\right)
$$




\section{§3. Application to the Known Constants.}

7. Suppose that $F$ contains a known constant $\alpha_{0}$ of the $\alpha$-set and a known angle $w_{4}=b_{4} t+\beta_{4}$ in which (the more general case) $b_{4}$ is a function of $\alpha_{0}$ and $\beta_{4}$ belongs to the $\beta$ set. Then applying (6) successively to the various combinations of $\alpha_{0}, w_{4}$ with the arbitraries and using (7), (9) we obtain

$$
\begin{aligned}
& \frac{\partial}{\partial \alpha^{\prime}}\left[\alpha, w_{4}\right]-\frac{\partial}{\partial \alpha}\left[\alpha^{\prime}, w_{4}\right]=\frac{\partial}{\partial w_{4}}\left[\alpha, \alpha^{\prime}\right]=0, \\
& \frac{\partial}{\partial \alpha^{\prime}}\left[\alpha, \alpha_{0}\right]-\frac{\partial}{\partial \alpha}\left[\alpha^{\prime}, \alpha_{0}\right]=\frac{\partial}{\partial \alpha_{0}}\left[\alpha, \alpha^{\prime}\right]=0, \\
& \frac{\partial}{\partial \alpha}\left[w, w_{4}\right]-\frac{\partial}{\partial w}\left[\alpha, w_{4}\right]=\frac{\partial}{\partial w_{4}}[w, \alpha]=0, \\
& \frac{\partial}{\partial \alpha}\left[\alpha_{0}, w\right]=\frac{\partial}{\partial \alpha_{0}}[\alpha, w]-\frac{\partial}{\partial w}\left[\alpha, \alpha_{0}\right], \\
& \frac{\partial}{\partial \alpha}\left[\alpha_{0}, w_{4}\right]=\frac{\partial}{\partial \alpha_{0}}\left[\alpha, w_{4}\right]-\frac{\partial}{\partial w_{4}}\left[\alpha, \alpha_{0}\right] .
\end{aligned}
$$

The first three of these give

$$
\left[\alpha_{i}, w_{4}\right]=\frac{\partial C_{4}}{\partial \alpha_{i}}, \quad\left[\alpha_{i}, \alpha_{0}\right]=\frac{\partial C_{0}}{\partial \alpha_{i}}, \quad\left[w_{i}, w_{4}\right]=\frac{\partial C_{4}}{\partial w_{i}} \quad(i=1,2,3),
$$

and the last two then give

$$
\left[\alpha_{0}, w_{i}\right]=\frac{d c_{i}}{d \alpha_{0}}-\frac{\partial C_{0}}{\partial w_{i}}, \quad \cdot\left[\alpha_{0}, w_{4}\right]=\frac{\partial C_{4}}{\partial \alpha_{0}}-\frac{\partial C_{0}}{\partial w_{4}} \quad(i=1,2,3),
$$

in which $C_{4}, C_{0}$ are, owing to the forms of the square brackets as deduced from the expressions for the coördinates, trigonometric series with the arguments $w_{i}$, $w_{4}$ and with coefficients depending on $\alpha_{i}, \alpha_{0} ; C_{4}$ consists of cosines only, $C_{0}$ of sines only. Moreover, $C_{4}$ is only defined by its derivates with respect to $\alpha_{0}$, $\alpha_{i}, w_{i}$ and may therefore contain terms independent of them, while $C_{0}$ is only defined by its derivatives with respect to $\alpha_{i}, w_{i}, w_{4}$ and might contain terms independent of them. The suppositions of $\S 1$ prevent the presence of such terms, so that $C_{0}, C_{4}$ are fully defined.

If the solution of the original equations has been obtained algebraically we have then sufficient material to find $C_{4}, C_{0}$. It may happen, however, that numerical values have been given to the constants in forming the solution, and for many purposes it is useful to possess equations for $C_{4}, C_{0}$ which will enable us to find their values in this case. It will be shown that they satisfy ordinary differential equations of the first order. 
These equations may be obtained directly from the results of arts. $3,4,7$ without using the original equations of motion. But as they involve $\partial^{\prime} F / \partial \alpha_{0}$, $d^{\prime} F / \partial w_{4}$, it is much easier to obtain them directly from the results of arts. 4, 7 and the equations of motion. This latter method, which also furnishes the vis viva equation, will be followed.

In this section, unless explicitly stated otherwise, the suffixes $i, j$ receive only the values $1,2,3$, that is, the letters to which they are attached are arbitraries of the solution.

8. The Vis Viva equation. Since

$$
\begin{gathered}
{\left[\alpha_{i}, w_{j}\right]=\frac{d c_{j}}{d \alpha_{i}}, \quad\left[\alpha_{i}, w_{4}\right]=\frac{d C_{4}}{d \alpha_{i}},} \\
\frac{d Q}{d t}=\Sigma_{i} b_{i} \frac{d Q}{d w_{i}}+b_{4} \frac{d Q}{d w_{4}},
\end{gathered}
$$

where $Q$ contains the time only through its presence in the angles $w_{i}, w_{4}$, we have

$$
\Sigma_{j} b_{j} \frac{d c_{j}}{d \alpha_{i}}+b_{4} \frac{d C_{4}}{d \alpha_{i}}=S\left(\frac{\partial \dot{x}}{\partial \alpha_{i}} \dot{x}-\ddot{x} \frac{\partial x}{\partial \alpha_{i}}\right)=\frac{\partial}{\partial \alpha_{i}}(T-F)
$$

But $\Sigma_{j} b_{j} d c_{j}=-d B$. Therefore, since there are no terms independent of the arbitrary constants and $b_{4}$ is a known constant,

$$
T-F-b_{4} C_{4}=-B
$$

9. The equation for $C_{4}$. Differentiating (13) with respect to the time,

or

$$
b_{4} \frac{d C_{4}}{d t}=\frac{d}{d t}(T-F)=S(x \dot{x}-\ddot{x} \dot{x})-b_{4} \frac{d^{\prime} F}{d w_{4}},
$$

$$
\frac{d C_{4}}{d t}=-\frac{d^{\prime} F}{d w_{4}}
$$

The variable $C_{4}$ is therefore similar to the $L$ of M. Poincare in his paper Sur les équations de la lune. ${ }^{*}$ We might have defined $C_{4}$ by means of this equation and have then reversed the processes of this article and obtained the first and third of equations (11). As $C_{4}$ can contain a constant term, the definition is not complete, but will be made so by the equation obtained in art. 9 .

* Bulletin Astronomique, vol. 17 (1900), p. 167. 
10. The equation for $C_{0}$.-Multiply the first of equations (12) by $b_{i}$, the second by $b_{4}$ and form the sum. We obtain

$$
\begin{aligned}
\Sigma_{i} b_{i} \frac{d c_{i}}{d \alpha_{0}}+b_{i} \frac{\partial C_{4}}{\partial \alpha_{0}}-\frac{d C_{0}}{d t} & =S\left(\frac{\partial \dot{x}}{\partial \alpha_{0}} x-\ddot{x} \frac{\partial x}{\partial \alpha_{0}}\right) \\
& =\frac{\partial T}{\partial \alpha_{0}}-\frac{\partial F}{\partial \alpha_{0}}+\frac{\partial^{\prime} F}{\partial \alpha_{0}} \\
& =\frac{\partial}{\partial \alpha_{0}}\left(b_{4} C_{4}\right)-\frac{d B}{d \alpha_{0}}+\frac{\partial^{\prime} F}{\partial \alpha_{0}},
\end{aligned}
$$

by (13). In this equation $B$ is supposed to be expressed in terms of $\alpha_{i}, \alpha_{0}$. Let $d B_{c} / d \alpha_{0}$ denote the derivative of $B$ with respect to $\alpha_{0}$ when $B$ is expressed in terms of $c_{1}, c_{2}, c_{3}, \alpha_{0}$, so that

$$
\frac{d B}{d \alpha_{0}}=\frac{d B_{c}}{d \alpha_{0}}+\Sigma_{i} \frac{d B_{c}}{d c_{i}} \frac{d c_{i}}{d \alpha_{0}}=\frac{d B_{c}}{d \alpha_{0}}-\Sigma_{i} b_{i} \frac{d c_{i}}{d \alpha_{0}} .
$$

Substituting in the former equation and rearranging, we obtain

the equation required.

$$
\frac{a C_{0}}{d t}+C_{ \pm} \frac{d b_{4}}{d \alpha_{10}}-\frac{d B_{c}}{d \overline{\alpha_{0}}}=-\frac{\partial^{\prime} F}{\partial \alpha_{0}}
$$

Since $C_{0}$ contains no constant term, $(15)$ gives it completely when $C_{4}$ has been obtained. We already know $C_{4}$ from (14) except its constant term. But since $C_{0}$ contains no term proportional to the time, the constant term in $C_{4}$ is given by

$$
\frac{d b_{4}}{d \alpha_{0}}\left(C_{4}\right)^{0}-\frac{d B_{c}}{d \alpha_{0}}=-\left(\frac{\partial^{\prime} F}{\partial \alpha_{0}}\right)^{0}
$$

where $(\cdot)^{0}$ denotes the constant term in the expansion of the enclosed function in trigonometric series.

If $b_{4}$ does not depend on $\alpha_{0}$, it is easily seen that the equation has its second term absent and $C_{0}$ can be found independently of $C_{4}$.

But now the constant part of $C_{4}$ cannot be determined by $(15)^{0}$. The difficulty is turned by following the investigation of this article with $b_{4}$ as the constant instead of $\alpha_{0}$. Then as $b_{4}$ occurs explicitly in $F$ only through its presence in $w_{4}$ the equation (15) reduces to

giving

$$
\frac{d C_{0}}{d t}+C_{4}-\frac{d B_{c}}{d b_{4}}=0
$$

$$
\left(C_{4}\right)^{0}=\frac{d B_{c}}{d b_{4}}
$$

The $C_{0}$ of this equation is, of course, different from that of (15). 
There may be other known constants $\alpha_{p}$ present in $F$. If $b_{4}$ depends on $\alpha_{p}$ then there will be an equation for $C_{p}$ corresponding to (15). If $b_{4}$ is independent of $\alpha_{p}$, the equation satisfied by the corresponding $C_{p}$ is

$$
\frac{d C_{p}}{d t}-\frac{d B_{c}}{d \alpha_{p}}=-\frac{\partial^{\prime} F}{\partial \alpha_{p}}
$$

where $C_{p}$ consists of sines only. It will be convenient in what follows to suppose that any other constants are all of the latter type and therefore may either be treated as numerical constants in which case the corresponding equation (16) will be unnecessary, or as algebraic constants in which case it may be necessary to use the equation. Both cases may arise in the method of the variation of known constants which is developed below.

11. Suppose that $F^{\prime}$ is homogeneous of order $q$ with respect to $x, y, z$, and a certain constant $A$ present in $F$, there being also present an absolute constant (e. g., the "gravitation" constant) which restores the proper physical dimensions of the equations of motion.

Then we have, symbolically,

$$
F, B, T=q \text { (length). }
$$

The equations of motion show that

$$
\text { time }=\left(1-\frac{1}{2} q\right) \text { (length), }
$$

and since $\left[c_{i}, w_{i}\right]=1, b=-1$ (time),

$$
c_{i}=\left(1+\frac{1}{2} q\right)(\text { length })=\frac{q+2}{q-2}\left(b_{i}\right), \quad F, B, T=\frac{2 q}{q+2}\left(c_{i}\right) .
$$

The choice of $\alpha_{0}$ has been quite arbitrary. When it is a function of $b_{4}$ take it so that it is of the same dimensions as the $c_{i}$ and further such that

$$
\frac{d \alpha_{0}}{d b_{4}}=\frac{q+2}{q-2} \alpha_{0}
$$

As $A$ is merely defined as a length, choose it so that

$$
\frac{d A}{A}=\frac{2}{q+2} \frac{d \alpha_{0}}{\alpha_{0}} \text {. }
$$

Thus $A, \alpha_{0}$ are defined in terms of $b_{4}$, but the manner in which they occur in $F^{*}$ is still arbitrary except with respect to their dimensions.

Since $B$ will be homogeneous with respect to the $c_{i}, \alpha_{0}$ we have

$$
\alpha_{0} \frac{d B_{c}}{d \alpha_{0}}=\frac{2 q}{q+2} B-\Sigma_{i} c_{i} \frac{d B}{d c_{i}}=\frac{2 q}{q+2} B+\Sigma_{i} b_{i} c .
$$


12. Multiply (15) by $q+2$ and apply the results for $d A, d \alpha_{0}$ in art. 11 . We obtain

$$
-(q+2) \alpha_{0} \frac{d C_{0}}{d t}-(q-2) b_{4} C_{4}+(q+2) \alpha_{0} \frac{d B_{c}}{d \alpha_{0}}=2 A \frac{\partial^{\prime} F}{\partial A},
$$

where $F$ is supposed to be expressed in terms of $A$ instead of $\alpha_{0}$.

Now $F$ is homogeneous of order $q$ with respect to $x, y, z, A$. Hence, multiplying equations (1) by $2 x, 2 y, 2 z$, and adding their sum to the last equation, we obtain

$$
\frac{d^{2}}{d t^{2}} r^{2}-4 T-(q+2) \alpha_{0} \frac{d C_{0}}{d t}-(q-2) b_{4} C_{4}+(q+2) \alpha_{0} \frac{d B_{c}}{d \alpha_{0}}=2 q F,
$$

which, by (15) and the last result in art. 11, becomes

$\frac{d^{2}}{d t^{2}} r^{2}-(q+2) \alpha_{0} \frac{d C_{0}}{d t}-(q-2)(T-F+B)+2 q B$

or, reducing,

$$
+(q+2) \Sigma b_{i} c_{i}=2 q F+4 T,
$$

$$
T+F-B-b_{1} c_{1}-b_{2} c_{2}-b_{3} c_{3}=\frac{d}{d t}\left(\frac{1}{2+q} \frac{d}{d t} r^{2}-\alpha_{0} C_{0}\right) .
$$

Denote by zero index the constant terms in the expansion of these functions. Then, since the right-hand member contains no constant term,

$$
T^{0}+F^{0}=B+b_{1} c_{1}+b_{2} c_{2}+b_{3} c_{3},
$$

a result independent of $q$.

13. The equation (18) gives a useful formula for finding the $c_{i}$ when the solution has been obtained with any set of arbitraries and even when numerical values have been given to the $\alpha$-set. For

or

$$
\begin{gathered}
c_{i}=\frac{\partial}{\partial b_{i}}\left(B+\Sigma b_{i} c_{i}\right)=\frac{\partial}{\partial b_{i}}\left(T^{\dot{0}}+F^{0}\right) \\
=S\left[\dot{x} \frac{\partial \dot{x}}{\partial b_{i}}+\ddot{x} \frac{\partial x}{\partial b_{i}}\right]^{0}=S\left[\dot{x} \frac{d x}{d w_{i}}+\frac{d}{d t}\left(\dot{x} \frac{\partial x}{\partial b_{i}}\right)\right]^{0} .
\end{gathered}
$$

$$
c_{i}=\left[\dot{x} \frac{\partial x}{\partial w_{i}}+\dot{y} \frac{\partial y}{\partial w_{i}}+\dot{z} \frac{\partial z}{\partial w_{i}}\right]^{0}
$$

Define a new constant $c_{4}$ by a similar equation for $i=4$. We then have or by (18),

$$
b_{1} c_{1}+b_{2} c_{2}+b_{3} c_{3}+b_{4} c_{4}=2 T^{0},
$$

$$
T^{0}-F^{0}-b_{4} c_{4}=-B \text {. }
$$

Comparing with the vis viva equation, we obtain

$$
c_{4}=\left(C_{4}\right)^{0} \text {. }
$$




\section{§ 4. Canonical Equations of Motion.}

14. The equations (14), (15) may be written

$$
\frac{d C_{4}}{d t}=-\frac{\partial^{\prime} F}{\partial w_{4}}, \quad \frac{d}{d t}\left(C_{0}-t \frac{\partial B_{c}}{\partial \alpha_{0}}\right)=-\frac{\partial^{\prime} F}{\partial \alpha_{0}}-C_{4} \frac{d b_{4}}{d \alpha_{0}} .
$$

These with the equations of motion (1) suggest the following transformation. Put

$$
2 T=\dot{x}^{2}+\dot{y}^{2}+\dot{z}^{2}, \quad W_{0}=-C_{0}-b_{0} t+\beta_{0}, \quad \phi=T-F-b_{4} C_{4},
$$

where $\beta_{0}$ is a constant. If we consider $x, y, x, C_{4}, \alpha_{0}$ and $\dot{x}, \dot{y}, \dot{z}, w_{4}, W_{0}$ as independent variables and $\phi$ expressed as a function of them, the equations (1) and (21) may be written in the canonical form

$$
\left\{\begin{array}{c}
\frac{d x}{d t}=\frac{\partial \phi}{\partial x}, \quad \frac{d \dot{x}}{d t}=-\frac{\partial \phi}{\partial x} ; \cdots, \ldots ; \ldots, \ldots ; \\
\frac{d C_{4}}{d t}=\frac{\partial \phi}{\partial w_{4}}, \quad \frac{d w_{4}}{d t}=-\frac{\partial \phi}{\partial C_{4}} ; \\
\frac{d \alpha_{0}}{d t}=\frac{\partial \phi}{\partial W_{0}}, \quad \frac{d W_{0}}{d t}=-\frac{\partial \phi}{\partial \alpha_{0}} ;
\end{array}\right.
$$

for $\phi$ does not contain $W_{0}$ explicitly, $\alpha_{0}$ is a constant, and $d w_{4} / d t=b_{4}$. The same equations hold if $b_{4}$ is not a function of $\alpha_{0}$.

We may either use the first four or all five pairs of equations and the solutions of such canonical systems are well known. The last pair gives $\alpha_{0}=$ constant and defines $W_{0}$. It will be more convenient, however, to treat $\alpha_{0}$ as a given constant and use only the first four equations in the following work.

When (22) are solved canonically in terms of the constants $c_{j}$ and the angles $w_{j}(j=1,2,3,4)$ we find

$$
\phi=\text { constant }=-B^{\prime}, \quad b_{j}=-\frac{d B^{\prime}}{d c_{j}} .
$$

The previous articles show that $B^{\prime}=B+b_{4} c_{4}$ where $B$ is independent of $c_{4}$ [the assumption that the constant parts of $\phi$, etc., contain no parts independent of $c_{1}, c_{2}, c_{3}$ no longer holds]. Hence, as before, $b_{i}=-\partial B_{4} / \partial c_{i}$. Also the expressions for the coördinates are independent of $c_{4}$.

15. All the preceding investigations might have been developed by starting with these canonical equations and solving them by means of JACOBI's method, obtaining the results in the inverse order. I have preferred to follow the more natural if somewhat longer development as it seems to make the deduction of some of the results rather more clear in view of the applications which follow. 
If there be angles other than $w_{4}$ and constants other than $\alpha_{0}$ present in $F$, similar results may be obtained. In fact, the extension of this method to any number of variables, angles and constants will be sufficiently obvious. To each angle and constant corresponds respectively a pair of canonical equations; to $\phi$ must be added one term for each angle present. When this has been done, $\phi$, considered as a function of all the variables, is independent of $t$ explicitly and $\phi=$ const. is an integral of the equations.

\section{§5. Variation of Constants.}

16. Variation of arbitrary constants.-Let a new function $\psi$ be added to $\phi$, then, using the canonical set $c_{1}, c_{2}, c_{3}, c_{4}, w_{1}, w_{2}, w_{3}, w_{4}$, the equations satisfied by them are

$$
\frac{d c_{j}}{d t}=-\frac{\partial}{\partial w_{j}}\left(\psi-B^{\prime}\right)=-\frac{\partial \psi}{\partial w_{j}}, \quad \frac{d w_{j}}{d t}=\frac{\partial}{\partial c_{j}}\left(\psi-B^{\prime}\right) \quad(j=1,2,3,4),
$$

where $\psi$ and $B^{\prime}$ are expressed in terms of $c_{j}, w_{j}$, of the given constants and the time, $B^{\prime}$ being a function of the $c_{j}$ and the constants present in $\phi$.

The method appears to fail if $\psi$ contains none of the arbitraries $c_{j}, w_{j}$. But the equations of motion depend on the derivatives of $\psi$ with respect to the variables; we can therefore assume that $\psi$ is not independent of the arbitraries.

If $\psi$ is independent of the $w_{j}$, the $c_{i}$ are constant and the equations for $w_{j}$ are easily solved. If $\psi$ contains a small factor $\alpha$ and a term with argument $b t+\beta$, where $b$ is small and $\beta$ independent of the $\beta_{i}$, the corresponding terms in $w_{i}$ will contain divisors $b, b^{2} / \alpha, b^{3} / \alpha^{2} ; \ldots$; when $\beta$ is not independent of $\beta_{j}$ the first divisor will in general be $b^{2}$.

17. Variation of the given constants. Let $\psi$ be due to a small addition $\delta \alpha_{0}$ to $\alpha_{0}$, one of the constants present in $F$ where $\delta \alpha_{0}$ may be a constant or a function of the time. Then using the canonical equations (22) and assuming that $\delta a_{0}$ produces variations $\delta c_{j}, \delta w_{j}$ and that squares of the variations may be neglected,

$$
\psi=\Sigma_{j}\left(\frac{\partial \phi}{\partial c_{j}} \delta c_{j}+\frac{\partial \phi}{\partial w_{j}} \partial w_{j}+\frac{\partial \phi}{\partial \alpha_{0}} \delta \alpha_{0}\right)=-\Sigma_{j} \frac{d B^{\prime}}{d c_{j}} \delta c_{j}-\frac{d B^{\prime}}{d \alpha_{0}} \delta \alpha_{0},
$$

since $B^{\prime}$ is independent of the $w_{j}$.

The equations of variations are

$$
\begin{gathered}
\frac{d}{d t} \delta c_{j}=-\frac{\partial \psi}{\partial w_{j}}=\Sigma_{j^{\prime}} \frac{d B^{\prime}}{d c_{j^{\prime}}} \frac{\partial}{\partial w_{j}} \delta c_{j^{\prime}}+\frac{d B^{\prime}}{d \alpha_{0}} \frac{\partial}{\partial w_{j}} \delta \alpha_{0}, \\
\frac{d}{d t}\left(w_{j}+\delta w_{j}\right)=\frac{\partial \psi}{\partial c_{j}}+b_{j}=-\frac{\partial}{\partial c_{j}}\left(\Sigma_{j^{\prime}} \frac{d B^{\prime}}{d c_{j^{\prime}}} \delta c_{j^{\prime}}+\frac{d B^{\prime}}{d \alpha_{0}} \delta \alpha_{0}\right)+b_{j}, \begin{array}{l}
\left(j, j^{\prime}=1,2,3,4\right) .
\end{array}
\end{gathered}
$$


As squares of the variations are neglected we can substitute constant values for the coefficients of the variations in the right hand members and therefore

$$
\begin{gathered}
\frac{d}{d t} \delta c_{j}=-\Sigma_{j^{\prime}} b_{j^{\prime}} \frac{\partial}{\partial w_{j}} \delta c_{j^{\prime}}+\frac{d B^{\prime}}{d \alpha_{0}} \frac{\partial}{\partial w_{j}} \delta x_{0}, \\
\frac{d}{d t}\left(w_{j}+\delta w_{j}\right)=\frac{\partial}{\partial c_{j}}\left(\Sigma_{j^{\prime}} b_{j^{\prime}} \delta c_{j^{\prime}}-\frac{d B^{\prime}}{d \alpha_{0}} \delta \alpha_{0}\right)+b_{j} .
\end{gathered}
$$

18. Suppose that $\delta \alpha_{0}$ is independent of the $w_{j}, c_{j}$. The last terms of the equations for $\delta c_{j}$ then vanish and they are satisfied by

$$
\delta c_{j}=0 \text {. }
$$

Substituting in the second set we find, since $B^{\prime}=B+b_{4} c_{4}$,

$$
\frac{d}{d t}\left(w_{j}+\delta w_{j}\right)=\frac{d b_{j}}{d \alpha_{0}} \delta \alpha_{0}+b_{j}
$$

That is, in order to obtain the new value of $w_{j}$, all that is necessary is to substitute $\alpha_{0}+\delta \alpha_{0}$ for $\alpha_{0}$ in the expression for $b_{j}$ expressed as a function of $c_{1}, c_{2}, c_{3}, \alpha_{0}$.

Suppose that the original solution had been expressed in terms of any arbitraries $\alpha_{1}, \alpha_{2}, \alpha_{3}$ of the $\alpha$-set, and of the known constant $\alpha_{0}$; the $c_{i}$ having been obtained in terms of them by the method of art. 13. Then the variations of $\alpha_{i}$ are found by solving the equations

$$
\frac{d c_{i}}{d \alpha_{1}} \delta \alpha_{1}+\frac{d c_{i}}{d \alpha_{2}} \delta \alpha_{2}+\frac{d c_{i}}{d \alpha_{3}} \delta \alpha_{3}=-\frac{d c_{i}}{d \alpha_{0}} \delta \alpha_{0} \quad(i=1,2,3),
$$

for $\delta \alpha_{1}, \delta \alpha_{2}, \delta \alpha_{3}$. The changes in the coefficients of the periodic terms are obtained by substituting these changes of the constants therein. But the changes in the $b_{i}$ are given by

$$
\delta b_{i}=\frac{d b_{i}}{d \alpha_{1}} \delta \alpha_{1}+\frac{d b_{i}}{d \alpha_{2}} \delta \alpha_{2}+\frac{d b_{i}}{d \alpha_{3}} \delta \alpha_{3}+\frac{d b_{i}}{d \alpha_{0}} \delta \alpha_{0} \quad(i=1,2,3),
$$

that is to say, we must also substitute the change of $\alpha_{0}$ as well as those of $\alpha_{1}, \alpha_{1}, \alpha_{3}$ in the $b_{i}$.

The same results hold if $b_{4}$ is not a function of $\alpha_{0}$.

19. If $\psi$ be due to a variation $\delta w_{4}$ of $w_{4}$, we have in a similar manner,

$$
\psi=\Sigma_{j} b_{j} \delta c_{j}
$$

and the equations of variations are

$$
\frac{d}{d t} \delta c_{j}=-\Sigma_{j^{\prime}} b_{j^{\prime}} \frac{\partial}{\partial w_{j}} \delta c_{j^{\prime}}, \quad \frac{d}{d t}\left(w_{j}+\delta w_{j}\right)=-\frac{\partial}{\partial c_{j}}\left(\Sigma_{j} \frac{d B^{\prime}}{d c_{j^{\prime}}} \delta c_{j^{\prime}}\right)+b_{j} .
$$


Suppose that the variation of $w_{4}$ is given. The left hand member of the last of the second set, namely,

¿s known.

$$
\frac{d}{d t} \delta w_{4}=\Sigma_{j^{\prime}} b_{j^{\prime}} \frac{\partial}{\partial c_{4}} \delta c_{j^{\prime}}
$$

The value of $\delta w_{4}$ may consist of periodic and non-periodic terms: suppose it to be independent of the angles $w_{1}, w_{2}, w_{3}$ and of $c_{1}, c_{2}, c_{3}$. Let us try if $\delta c_{1}=0, \delta c_{2}=0, \delta c_{3}=0$ and $\delta c_{4}$ independent of $w_{1}, w_{2}, w_{3}$ can satisfy the equations. The first three of the first pair are satisfied and the others become

$$
\frac{d}{d t} \delta c_{4}=-b_{4} \frac{\partial}{\partial w_{4}} \delta c_{4} \quad \frac{d}{d t} \delta w_{4}=b_{4} \frac{\partial}{\partial c_{4}} \delta c_{4}, \quad \frac{d}{d t} \delta w_{i}=b_{4} \frac{\partial}{\partial c_{i}} \delta c_{4} \quad(i=1,2,3) .
$$

If $\delta w_{4}=q t+q^{\prime}$, where $q, q^{\prime}$ are given constants, the equations are satisfied by $\delta c_{4}=q c_{4} / b_{4}, \delta w_{i}=0$. It does not appear to be possible in general to satisfy the equations with other values for $\delta w_{4}$.

\section{§6. Applications to the Lunar Theory.}

20. The results contained in the previous sections are more general in their character than is necessary for the applications to most problems in celestial mechanics and in particular for that one which is known as the lunar theory. Without going into details it is sufficient to state here that the few restrictions imposed are satisfied in these applications.

Let $x, y, z, r$ denote the coördinates and distance of the moon relatively to the earth's center; $x^{\prime}, y^{\prime}, z^{\prime}, r^{\prime}$, those of the sun relatively to the center of mass of the earth and moon ; $\mu=E+M$, the sum of the masses of the earth and moon; $m^{\prime}$ the mass of the sun; let the three bodies be considered to attract in the same way as particles of the same masses situated at their centers of mass respectively. Also suppose that the motion of the sun is known and that all other influences are neglected. Then it is well known that

$$
F=\frac{\mu}{r}+m^{\prime}\left\{r^{2}-2 r\left(x x^{\prime}+y y^{\prime}+z z^{\prime}\right)+{r^{\prime 2}}^{-\frac{1}{2}}-m^{\prime}\left(x x^{\prime}+y y^{\prime}+z z^{\prime}\right){r^{\prime-3}}^{3},\right.
$$

where, after the substitution of polar coördinates for $x^{\prime}, y^{\prime}, z^{\prime}$ and expansion in powers of $1 / r^{\prime}$, the term $1 / r^{\prime 3}$ is dropped and the coefficient of $\left(r^{\prime}\right)^{-p}$ is multi. plied by

We can therefore put

$$
\kappa_{p}=\left(\frac{E}{E+M}\right)^{p-1}-\left(\frac{-M}{E+M}\right)^{p-1} .
$$

$$
F=\frac{\mu}{r}+m^{\prime} \sum_{p=2}^{\infty} \kappa_{p} R_{p}^{\prime}\left(r^{\prime}\right)^{-p-1},
$$

where $R_{p}^{\prime}$ is a homogeneous function of order $p$ with respect to $x, y, z$, and also 
contains trigonometric functions of the angular position of the sun. Further, if $n^{\prime}, a^{\prime}$ be the mean motion and mean distance of the sun, $m^{\prime}+\mu={n^{\prime 2}}^{\prime 3}$, or, putting $\lambda=\mu /\left(m^{\prime}+\mu\right)$,

$$
m^{\prime}=n^{\prime 2} a^{\prime 3}(1-\lambda) \text {. }
$$

We shall now suppose that the plane of the sun's orbit is fixed and that its motion can always be expressed in terms of $n^{\prime}, e^{\prime}$ and the angle $n^{\prime} t+\beta_{4}$; when its motion is elliptic $n^{\prime}, \beta_{4}, e^{\prime}$ are constants. Properly, there are two angles $n^{\prime} t+\epsilon^{\prime}, n^{\prime} t+\epsilon^{\prime}-\pi^{\prime}$, but well known results show that we may treat them as though $\pi^{\prime}$ were zero and they also indicate where $\pi^{\prime}$ has to be inserted in the final results. Thus we can put

$$
F=\frac{\mu}{r}+{n^{\prime 2}}^{2}(1-\lambda) \sum_{p=2}^{\infty} \frac{R_{p}}{{a^{\prime \prime}}^{-2}},
$$

where $R_{p}$ is homogeneous and of order $p$ relatively to $x, y, z$ and contains $\kappa_{p}$, $e^{\prime}$ and trigonometric functions of $n^{\prime} t+\epsilon^{\prime}$.

21. We have from the equations of motion,

$$
\frac{1}{2} \frac{d^{2}}{d t^{2}} r^{2}-2 T=S x \frac{\partial F}{\partial x}=-\frac{\mu}{r}+{n^{\prime}}^{2}(1-\lambda) \Sigma_{p} p R_{p}\left(a^{\prime}\right)^{-p+2} \quad(p=2,3, \cdots) .
$$

Eliminating $T$ between this equation and (17) we find, since $q=-1$ here,

$$
\frac{3}{2} \frac{\mu}{r}-\frac{1}{2} n^{\prime 2}(1-\lambda) \Sigma_{p}(p-2) R_{p}\left(a^{\prime}\right)^{-p+2}-B-\Sigma_{i} b_{i} c_{i}=\frac{3}{4} \frac{d^{2}}{d t^{2}} r^{2}-\alpha_{0} \frac{d C_{0}}{d t}
$$

The right hand member contains no constant term. Hence

$$
\text { (25) } B+b_{1} c_{1}+b_{2} c_{2}+b_{3} c_{3}=\frac{3}{2}\left(\frac{\mu}{r}\right)^{0}-\frac{1}{2} n^{\prime 2}(1-\lambda) \Sigma_{p}(p-2)\left(R_{p}\right)^{0}\left(a^{\prime}\right)^{-p+2} \text {. }
$$

The equations (14), (15) show that $d C_{0} / d t$ is a quantity whose lowest order is $n^{\prime 2} / \alpha_{0}$. As it is usual in the lunar theory to expand in powers of $n^{\prime}$ and $1 / a^{\prime}$, it will be more convenient to put $\alpha_{0} C_{0}=C_{0}^{\prime}$ so that $d C_{0}^{\prime} / d t$ is a quantity whose lowest order is ${n^{\prime 2}}^{2}$ and is expansible like the coördinates. We therefore have

$$
\begin{array}{r}
\frac{d C_{0}^{\prime}}{d t}=\frac{3}{4} \frac{d^{2}}{d t^{2}}\left(r^{2}\right)-\frac{3}{2} \frac{\mu}{r}+\frac{1}{2} n^{\prime 2}(1-\lambda) \Sigma_{p^{\prime}}(p-2) R_{p^{\prime}}\left(a^{\prime}\right)^{-p+2}+f \\
(p=3,4, \cdots),
\end{array}
$$

$f$ being a constant so chosen as to make the constant part of the right-hand member zero.

22. The properties of equations (25), (26) which are remarkable and valuable for practical purposes arise principally in those investigations which permit us to neglect the ratio of the parallaxes of the sun and moon. This ratio is 
about $\frac{1}{0_{0}}$ and for most of the purposes which require the value of $C_{0}^{\prime}$, i. e., in the calculation of the indirect action of a planet (art. 24 below), it is possible to neglect it. But of the $R_{p}$, the first term $R_{2}$ is the only one which is independent of it and the factor $p-2$ causes $R_{2}$ to disappear in both the equations. The equation (26) then reduces to

$$
\frac{d C_{0}^{\prime}}{d t}=\frac{3}{4} \frac{d^{2}}{d t^{2}}\left(r^{2}\right)-\frac{3}{2}\left(\frac{\mu}{r}\right)+\frac{3}{2}\left(\frac{\mu}{r}\right)^{0} .
$$

Another observation may be made. If the object in view is such that we only require those terms in $C_{0}^{\prime}$ which are of long period relatively to the month, i. e., those in which the coefficients of $t$ in the arguments are small, the first term of the right-hand member will give rise to coefficients which are very small compared with those arising from the second member; in such cases we can generally put

$$
\frac{d C_{0}^{\prime}}{d t}=-\frac{3}{2}\left(\frac{\mu}{r}\right)+\frac{3}{2}\left(\frac{\mu}{r}\right)^{0},
$$

without committing sensible errors.

23. In nearly all cases of the indirect action of a planet, we can neglect the square of the ratio of the parallaxes. But it is well known that the constant parts of the functions we have been dealing with are expansible in powers of the square of this ratio. Hence neglecting the square of the ratio of the parallaxes,

$$
B+b_{1} c_{1}+b_{2} c_{2}+b_{3} c_{3}=\frac{3}{2}\left(\frac{\mu}{r}\right)^{0} .
$$

This is the result which I obtained in an earlier paper * and which I used to prove ADAMs' well known theorems and to obtain the secular accelerations directly from the expression for the parallax of the moon. It is true that the square of the ratio of the parallaxes was neglected, but it was easy to see from the few terms which Delaunay gave in a literal form that they could exert no sensible effect on the result. If doubts might still arise, owing to slow convergence, it is not difficult to remove them by means of the numerical results of my lunar theory taken in conjunction with equation (.25).

The methods used here differ in form from those which were adopted in the paper just referred to. The essential feature of the earlier method was really that of Delaunay, that is to say, the elliptic orbit was used as a first approximation and the variation of arbitrary constants applied to it somewhat in DeLAUNAY's manner. Here we are theoretically quite independent of the elliptic orbit, and any set of arbitrary constants which possesses the property of enabling

* See footnote, p. 334 above. 
us to expand in trigonometric series may be used. This has the practical advantage of permitting the calculation of $c_{1}, c_{2}, c_{3}$, by means of art. 13 from the results of any theory in whatever form it may be expressed and whatever be the arbitrary constants used. Provisionally it may be best to except the theory of HANSEN, although it is probable that the expression $\dot{x} \delta x+\dot{y} \delta y+\dot{z} \delta z$, which is the principal part of equation (19), might be transformed so as to be used in this case also without serious difficulty.

24. The results of arts. 15, 16 include Newcomb's theorem concerning the secular accelerations and the indirect action of a planet* as a particular case. In this valuable memoir he considered the action of a planet as a case of the disturbed motion of three bodies, that is to say the problem of the relative motion of the earth, moon and sun is considered to have been solved and the question was then to find out what variable values the 12 arbitrary constants which arise in the solution must have in order that the solution may retain the same form when another disturbing cause acts. He showed that if we first consider the orbit of the sun as elliptic and if the action of a planet causes variations $\delta e^{\prime}, \delta n^{\prime}$ in $e^{\prime}, n^{\prime}$, then the new non-periodic parts of $n, \pi_{1}, \theta_{1}$ (the mean motions of the moon, its perigee and node) are found by solving the equations

$$
\frac{\partial c_{i}}{\partial n} \delta n+\frac{\partial c_{i}}{\partial e} \delta e+\frac{\partial c_{i}}{\partial \gamma} \delta \gamma+\frac{\partial c_{i}}{\partial n^{\prime}} \delta n^{\prime}+\frac{\partial c_{i}}{\partial e^{\prime}} \delta e^{\prime}=0 \quad(i=1,2,3)
$$

for $\delta n, \delta e, \delta \gamma$, regarding $\delta n^{\prime}, \delta e^{\prime}$ as known, and substituting the variable values of $n, e, \gamma, n^{\prime}, e^{\prime}$ in the expressions of $n, \pi_{1}, \theta_{1}$ in terms of these five quantities. He remarks, "This curious theorem may embody some principle applicable to the disturbed motion of three bodies, which has not yet been fully mastered."

It appears from the investigation of this paper that the theorem is independent of the particular form in which the solar coördinates enter into the lunar disturbing function and further, that it is true for any of the constants which may be present in this disturbing function, provided always that the given variations of the known constants are independent of the lunar elements. Finally, it is applicable to any dynamical problem in which the coördinates satisfy the specified conditions and to any constants which may enter into the force,function in the manner specified at the outset.

HAVERFord COLlege, April 20, 1903.

* Action of the Planets on the Moon, IVashington Astronomical Papers, vol. 5 (1894), p. 191. 